\title{
Applications of Nanostructured Lipid Carriers: Recent Advancements and Patent Review
}

\author{
Neha Kanojia ${ }^{1(\mathbb{D})}$, Neelam Sharma ${ }^{1}$ (D), Nidhi Gupta ${ }^{1}{ }^{(\mathbb{D})}$, Sukhbir Singh ${ }^{1, *}$ (D) \\ 1 Chitkara College of Pharmacy, Chitkara University, Punjab, India \\ * Correspondence: singh.sukhbir12@gmail.com;
}

Scopus Author ID 56402098100

Received: 25.02.2021; Revised: 28.03.2021; Accepted: 3.04.2021; Published: 20.04.2021

\begin{abstract}
Nanostructured lipid carriers (NLCs) are a nano-particulate carrier system consisting of solid lipids, liquid lipids, emulsifying agents, and water. NLCs have gained continuous significance in recent times and have displayed tremendous drug delivery benefits against conventional dosage compositions. NLCs have significant prospects in the pharmaceutical and food industries. Its pharmaceutical application represents a wide spectrum of conditions such as hypertension, diabetes, Parkinsonism, epilepsy, hyperlipidemia, cancer, alopecia, hormone deficiency, topical inflammation, ocular, hepatic, and fungal diseases. This review briefly describes nanostructured lipid carriers in terms of their production techniques, characterization, recent advancements in pharmaceutical applications, and functional food delivery. This study also presented a review of recent patents based on nanostructured lipid carriers.
\end{abstract}

Keywords: food industries; nanostructured lipid carriers; pharmaceutical application; production techniques; recent patents.

(C) 2021 by the authors. This article is an open-access article distributed under the terms and conditions of the Creative Commons Attribution (CC BY) license (https://creativecommons.org/licenses/by/4.0/).

\section{Introduction}

Nanostructured lipid carriers (NLCs) are a nano-particulate carrier system derived from oil-in-water type nano-emulsions. Its key ingredients are lipid, emulsifying agents, and water. The lipid phase contains both solid (fat) and liquid (oil) lipids at room temperature. The rationale of NLCs based formulation is to manufacture particles in which the oil is integrated into solid lipid core, leading to superior loading capacity and controlled drug release since the drug dissolves in oil and concurrently encapsulates in solid lipid. The advantages of NLCs include reduced polymorphic transition, little crystalline index, enhanced encapsulation efficiency, drug loading, physical stability, improved chemical stability, bioavailability, and the controlled release of encapsulated components [1-6]. The low bioavailability of many drugs and functional foods is indeed a major concern that needs to be addressed in an attempt to achieve successful treatment. Therefore, there is a demand to synthesize a medication carrier system that removes such issues. Nowadays, several nanocarriers have been gradually explored to enhance therapeutic effectiveness and sustained characteristics of drug release while addressing issues like low solubility and poor bioavailability. Possible reasons for low bioavailability are given in Figure 1 [7-14]. Nevertheless, these nanocarrier systems must be toxic-free, possess a sufficient medication loading capacity, and potential drug targeting and controlled release features. In recent times, nanostructured lipid carriers (NLC) are continuously gaining significance and have demonstrated numerous benefits in drug delivery 
over traditional dosage formulations [15-18]. The lipids used for the production of NLC are typically biocompatible and biodegradable lipids with minimal toxicity [19]. The NLC matrix contains a combination of liquid and solid lipids that renders the matrix quite imperfect to hold additional drug molecules. NLC matrix exists as solid at room or body temperature by monitoring of liquid lipid content. Owing to a solid matrix, NLCs will more effectively incapacitate drugs that prevent the entity from coalescing. NLCs have low toxicity, biodegradation, drug safety, slow-release, and the removal of organic solvents during manufacturing [15, 19-22].

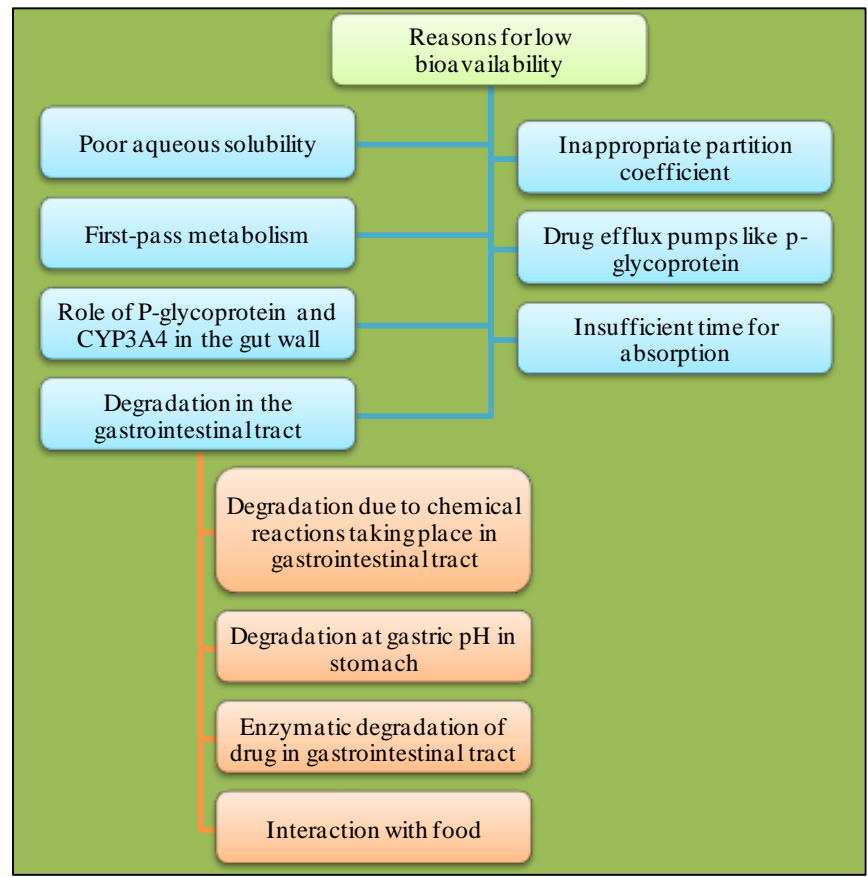

Figure 1. Reasons for low oral drug bioavailability.

NLCs have important prospects in the pharmaceutics and food industries. Its pharmaceutical application covers a wide range of disorders like hypertension, diabetes, Parkinsonism, epilepsy, hyperlipidemia, cancer, alopecia, hormone imbalance, topical inflammation, ocular, hepatic, and fungal diseases. This review briefly explains nanostructured lipid carriers concerning their production techniques, characterization techniques, recent application, and patents in pharmaceuticals and food delivery.

\section{Production Techniques of Nanostructured Lipid Carriers}

Several techniques for the production of NLC are given in Figure 2. Solventemulsification evaporation/diffusion and double-emulsion techniques are some of the usually preferred methodologies for manufacturing NLCs [23].

\subsection{Solvent-emulsification evaporation technique.}

This involves the dissolution of drug and solid and liquid lipids in water-immiscible organic solvents like cyclohexane and chloroform. This organic phase is dispersed into an aqueous phase containing emulsifiers to produce an o/w emulsion, which is followed by solvent evaporation under vacuum leading to the production of NLC due to precipitation of lipids in aqueous conditions (Figure 3). 


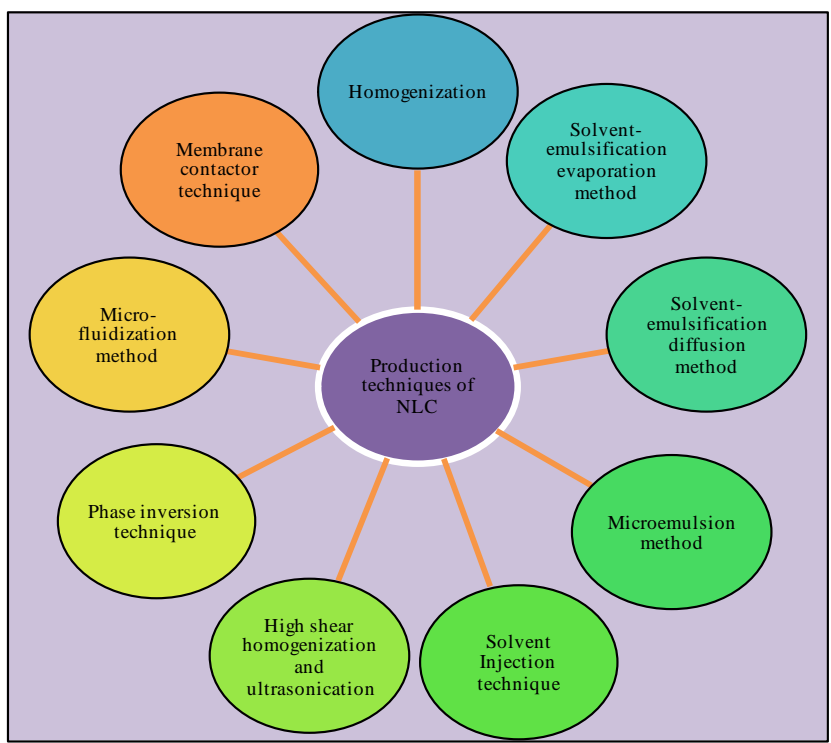

Figure 2. Production methodologies utilized for the production of nanostructured lipid carriers.

The advantages of this technique include avoidance of thermal stress, nevertheless, the utilization of organic solvent is a drawback [15, 24, 25].

\subsection{Solvent-emulsification diffusion technique.}

In this technique, the lipids and drug are dissolved in a water-saturated solvent, which is emulsified in a solution of emulsifier in solvent-saturated water phase using a homogenizer to create $\mathrm{o} / \mathrm{w}$ emulsion, which is further diluted with an excess of the aqueous phase, which causes organic solvent diffusion from emulsion droplets to aqueous phase leading to the production of NLC (Figure 3). The solvent removal could be through ultra-filtration or freezedrying. The solvent diffusion is a relatively preferred versatile technique as the employed solvents have a higher safety profile [15, 24, 25].

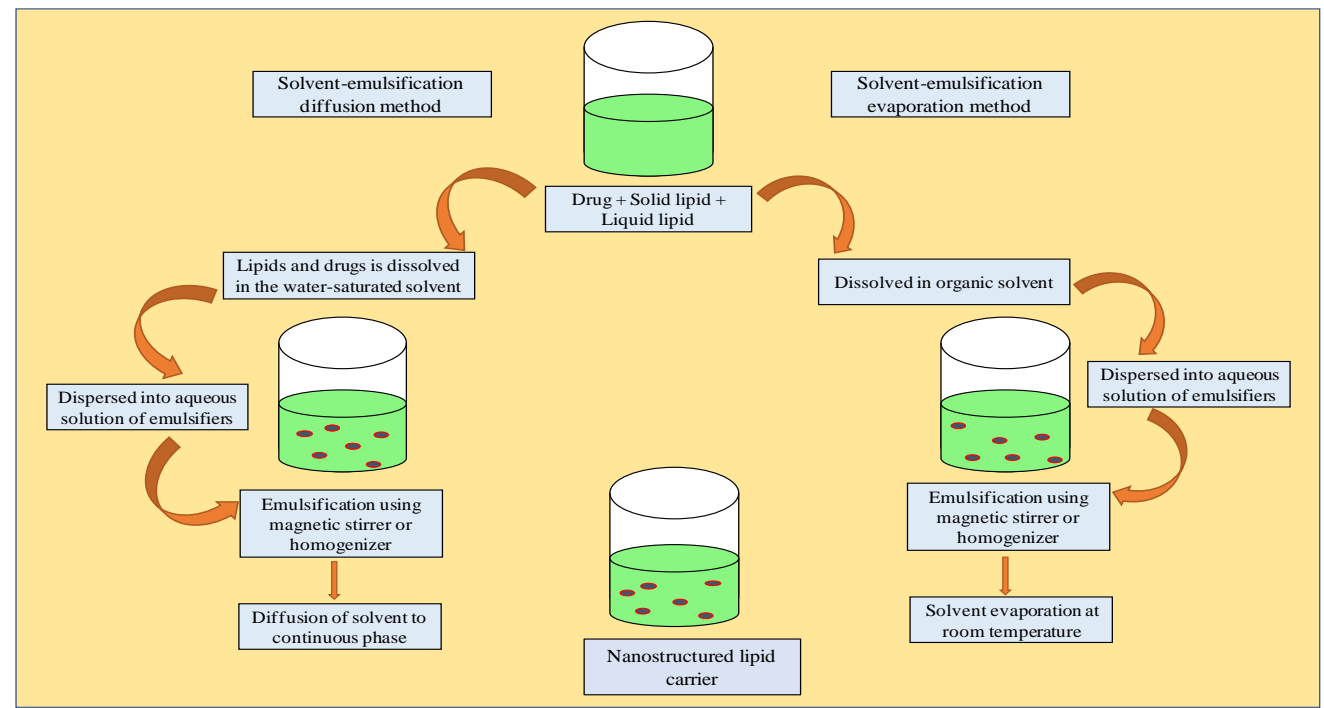

Figure 3. Schematic illustration for solvent-emulsification and solvent-diffusion evaporation techniques utilized for the production of nanostructured lipid carriers.

\subsection{Double emulsion technique.}

This approach is being used specifically to develop NLC of hydrophilic drugs to conquer the difficulty of hydrophilic moiety escape in the aqueous phase. An aqueous drug 
solution is transferred in molten lipid, subsequently dispersed into the aqueous emulsifier solution with sonication to produce w/o/w type double emulsion. Ultimately, NLCs will be collected through solvent evaporation and ultra-filtration (Figure 4) [15, 24, 25].

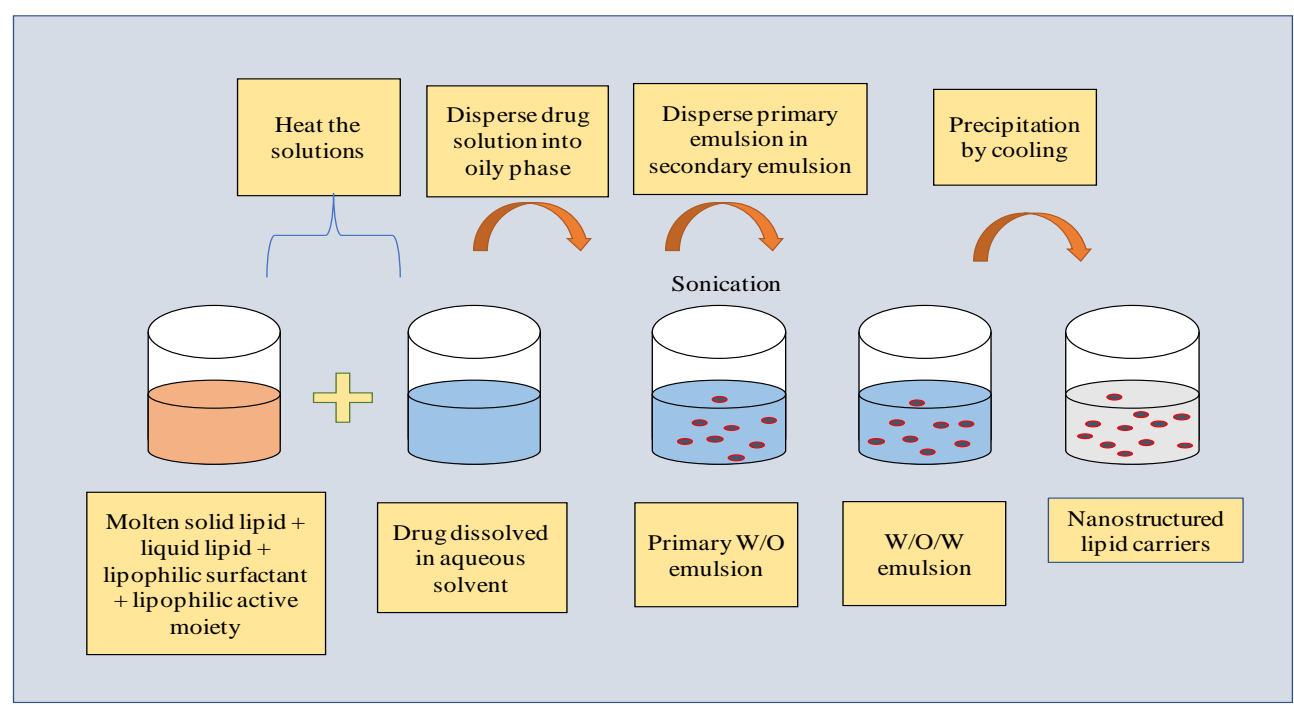

Figure 4. Schematic representation for double emulsion technique utilized for the production of nanostructured lipid carriers.

\subsection{Membrane contactor technique.}

It involves the melting of lipids beyond their melting point, which is filled into a pressurized container. Lipid melt is passed via a ceramic porous membrane to create tiny droplets. The aqueous solution passes tangentially inside the membrane under constant agitation, which pushes aside droplets produced at the pore's outlets. Cooling of formulation to ambient temperature tends to the production of NLC (Figure 5). This innovative technique's advantages are the industrial flexibility and monitoring of particle diameter through appropriate configured dimensions [15, 24, 25].

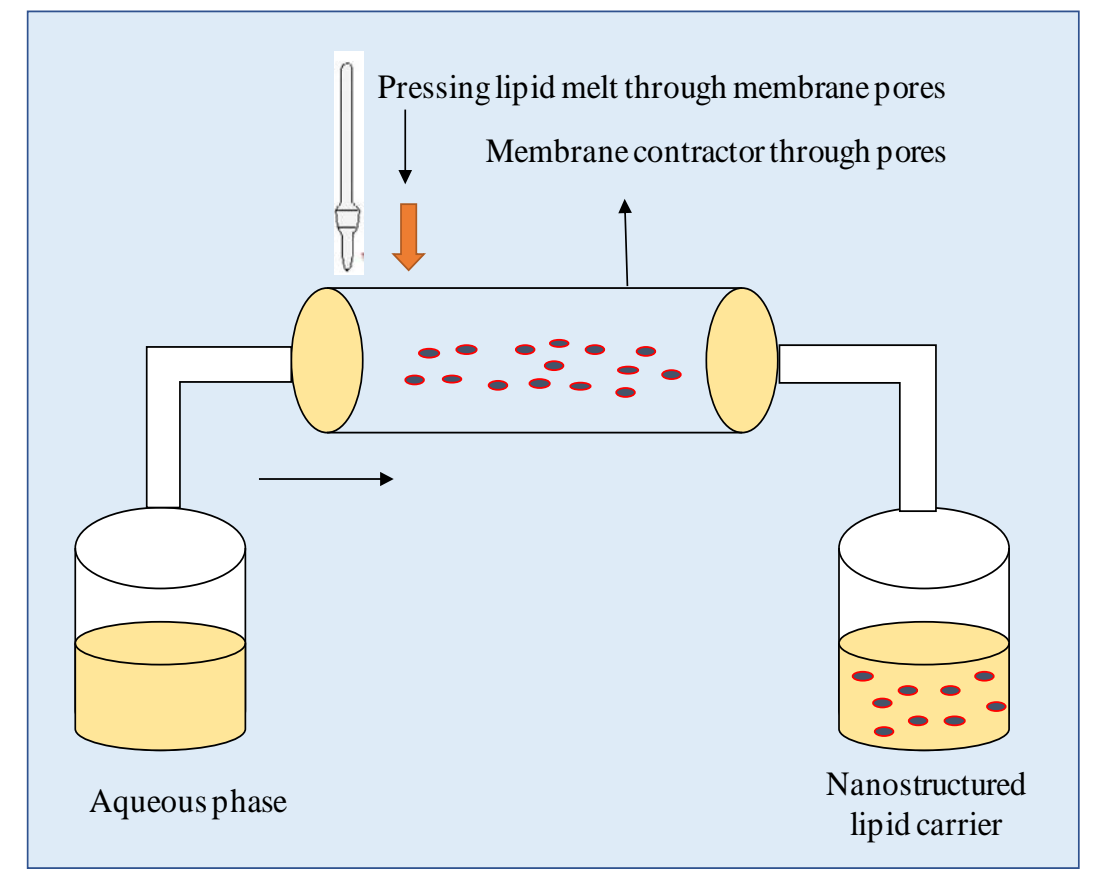

Figure 5. Schematic representation of membrane contractor technique utilized for the production of nanostructured lipid carriers. 


\section{Characterization Techniques for Nanostructured Lipid Carriers}

NLCs are characterized for particle size, zeta potential, morphology, entrapment efficiency, loading capacity, crystallinity or polymorphism, surface tension measurement, and additional colloidal structures [15]. This is necessary to ensure their efficiency, quality, and reliability. Several physicochemical characteristics of NLC, evaluation techniques, and their key features are described in Table 1.

Table 1. Techniques utilized for the characterization of nanostructured lipid carriers.

\begin{tabular}{|c|c|c|c|}
\hline Parameter & Technique & Key features & Ref. \\
\hline $\begin{array}{l}\text { Particle } \\
\text { measurement }\end{array}$ & $\begin{array}{ll} & \text { Photon correlation spectroscopy } \\
\circ & \text { Laser diffractometer } \\
\circ & \text { Field flow fractionation, dynamic } \\
& \text { ultra-microscopy } \\
\circ & \text { Ultrasonic spectroscopy } \\
\circ & \begin{array}{l}\text { Cryogenic transmission electron } \\
\text { microscopy analysis }\end{array} \\
\circ & \begin{array}{l}\text { Electro-acoustic } \\
\text { spectroscopy }\end{array} \\
\end{array}$ & $\begin{array}{l}\text { Polydispersity index is a measure of } \\
\text { particle homogeneity } \\
\text { (Range 0-1) }\end{array}$ & [26-29] \\
\hline Zeta potential & ○ Laser Doppler electrophoresis & $<-30 \mathrm{mV}$ or $>+30 \mathrm{mV}$ & [30] \\
\hline NLC morphology & $\begin{array}{ll}\circ & \text { Transmission and scanning } \\
\text { electron microscopy } \\
\circ & \text { Atomic force microscopy } \\
\circ & \text { Photon correlation spectroscopy } \\
\end{array}$ & $\begin{array}{l}\text { Dimensional and } \\
\text { structural characterization }\end{array}$ & [31-33] \\
\hline $\begin{array}{l}\text { Entrapment } \\
\text { efficiency }\end{array}$ & $\begin{array}{ll}\text { Ultrafiltration-centrifugation } \\
\text { method followed by } \\
\text { spectrophotometer }\end{array}$ & Determines encapsulated drug quantity & {$[27,34]$} \\
\hline Loading capacity & $\begin{array}{l}\text { Ultrafiltration-centrifugation } \\
\text { method followed by } \\
\text { spectrophotometer }\end{array}$ & $\begin{array}{l}\text { Quotient between entrapped drug to total } \\
\text { lipid weight }\end{array}$ & {$[27,34]$} \\
\hline $\begin{array}{l}\text { Crystallinity/ } \\
\text { polymorphism }\end{array}$ & $\begin{array}{ll} & \text { Differential scanning calorimetry } \\
\circ & \text { X-ray diffraction } \\
\end{array}$ & $\begin{array}{l}\text { Investigate crystallinity or polymorphic } \\
\text { structural changes }\end{array}$ & {$[27,35]$} \\
\hline $\begin{array}{l}\text { Surface tension } \\
\text { measurement }\end{array}$ & $\begin{array}{lll}\circ & \text { 'Platinum-Wilhelmy' plate } \\
\text { technique } & \\
\circ & \text { Contact angle determination } \\
\circ & \text { Kibron instrument } \\
\circ & \text { Du Noüy ring } \\
\end{array}$ & Measure surface tension of NLC & {$[36]$} \\
\hline \multirow{3}{*}{$\begin{array}{lr}\text { Evaluation } & \text { of } \\
\text { additional } & \text { colloidal } \\
\text { structures } & \end{array}$} & $\begin{array}{ll} & \text { Magnetic resonance investigation } \\
\circ & \text { Negative staining } \\
\end{array}$ & $\begin{array}{l}\text { Used to judge mobility } \\
\text { Give three-dimensional projections }\end{array}$ & {$[37,38]$} \\
\hline & $\circ \quad$ Electron spin resonance & $\begin{array}{l}\text { Direct as well as a non-invasive } \\
\text { assessment of spin-probe distribution } \\
\text { among lipid and aqueous }\end{array}$ & \\
\hline & ○ Raman spectroscopy & $\begin{array}{l}\text { Trace structural and chemical alterations } \\
\text { in vibrational transition }\end{array}$ & \\
\hline
\end{tabular}

\section{Recent Application of Nanostructured Lipid Carriers in Pharmaceuticals and Functional Food}

Recent pharmaceutical application of NLCSs encompasses various conditions like hypertension, diabetes, Parkinsonism, epilepsy, hyperlipidemia, cancer, alopecia, hormone deficiency, topical inflammation, ocular, hepatic, and fungal diseases. It also has broad applications in nutraceutical and functional foods. Hypertension is a severe medical disorder that dramatically increases the incidence of heart, brain, kidney, and other diseases. It is reported that one in six people globally, or almost one billion, would be influenced by hypertension, and this estimate is anticipated to expand to 1.5 million by the year 2025 [39]. Hypertension, in particular, is a considerable cardiovascular risk factor, and its incidence in diabetes mellitus would be raised. Diabetes mellitus is a category of metabolic disorders arising from insulin deficiency, insulin resistance, or sometimes both leading to chronic hyperglycemia with abnormal carbohydrate, fat, and protein metabolism [40]. Diabetes is affecting an 
estimated 30.3 million communities in the United States, while hypertension is reported at 73.6 percent of individuals aged 18 years or older $[41,42]$. NLCs based drug delivery systems could effectively improve the therapeutic efficacy of medications utilized to treat such fatal disorders and other disease conditions. The recent progression in the application of NLCs in the field of pharmaceuticals and food delivery is described in Table 2.

Table 2. Application of nanostructured lipid carriers in the delivery of functional food and pharmaceuticals.

\begin{tabular}{|c|c|c|c|c|}
\hline Drug & Purpose & Excipients & Technique & Ref. \\
\hline \multicolumn{5}{|c|}{ Hypertension } \\
\hline $\begin{array}{l}\text { Lercanidipine } \\
\text { hydrochloride }\end{array}$ & 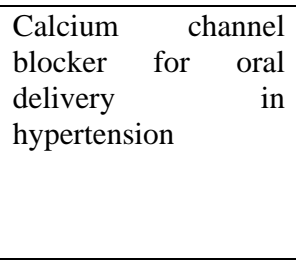 & $\begin{array}{l}\text { Glyceryl monostearate, } \\
\text { Stearic acid, Beeswax, } \\
\text { Carnauba wax, Labrafil M } \\
\text { 2130, Compritol ATO888, } \\
\text { Gelucire 44/14, Gelucire } \\
\text { 50/13, Span 80, Tween 80, } \\
\text { Lutrol@F68 }\end{array}$ & $\begin{array}{l}\text { Ultra-sonication and } \\
\text { emulsion } \\
\text { evaporation }\end{array}$ & [43] \\
\hline Carvedilol & $\begin{array}{lr}\beta- & \text { adrenoceptor } \\
\text { blocker for } & \text { oral } \\
\text { delivery } & \text { in } \\
\text { hypertension } & \\
\end{array}$ & $\begin{array}{l}\text { Gelucire, Cetyl palmitate, } \\
\text { Compritol 888, Tripalmitin, } \\
\text { Linseed oil, Tween } 80\end{array}$ & Probe sonication & [44] \\
\hline Sildenafil Citrate & $\begin{array}{lr}\text { Non-selective } & \\
\text { vasodilating } & \text { action } \\
\text { pulmonary arterial } \\
\text { hypertension }\end{array}$ & $\begin{array}{l}\text { Precirol, Beeswax, Stearic } \\
\text { acid } \\
\text { Polyvinyl alcohol }\end{array}$ & $\begin{array}{l}\text { Modified melt } \\
\text { emulsification }\end{array}$ & [45] \\
\hline Isradipine & $\begin{array}{l}\text { Oral delivery for } \\
\text { hypertension }\end{array}$ & 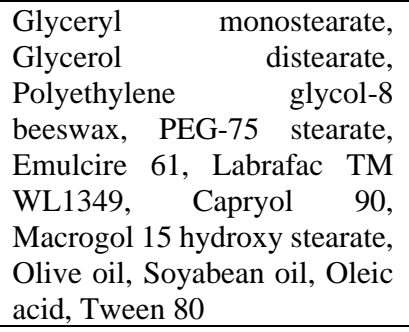 & $\begin{array}{l}\text { Solvent evaporation } \\
\text { and probe sonication }\end{array}$ & [46] \\
\hline \multicolumn{5}{|c|}{ Diabetes } \\
\hline Berberine & Hypoglycemic & $\begin{array}{l}\text { Pol Precirol® ATO5, Tween } \\
\text { 80, Oleic acid }\end{array}$ & $\begin{array}{l}\text { Hot Melt } \\
\text { homogenization }\end{array}$ & [47] \\
\hline Glibenclamide & Anti-diabetic & $\begin{array}{l}\text { Carbopol 934, Glyceryl } \\
\text { monostearate, Tween } 80, \\
\text { Triethanolamine }\end{array}$ & $\begin{array}{l}\text { Emulsion-solvent } \\
\text { diffusion } \\
\text { evaporation }\end{array}$ & [48] \\
\hline Insulin & Anti-diabetic & $\begin{array}{l}\text { Methocel A15C, Methocel } \\
\text { A4M, Soya lecithin, Tween } 80\end{array}$ & Solvent-evaporation & [49] \\
\hline \multicolumn{5}{|c|}{ Multiple disorders of central/peripheral nervous systems and peripheral organs } \\
\hline Rimonabant & $\begin{array}{l}\text { Intranasal delivery of } \\
\text { Cannabinoid } \\
\text { antagonist }\end{array}$ & 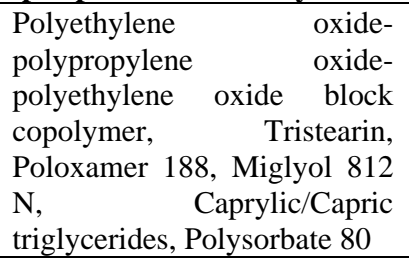 & $\begin{array}{l}\text { Melt and ultra- } \\
\text { sonication }\end{array}$ & [50] \\
\hline Bromocriptine & Anti-parkinsonism & $\begin{array}{l}\text { Lutrol F 68, Rylo MG 19, } \\
\text { Miglyol 812, Pluronic F127, } \\
\text { glyceryl monooleate, } \\
\text { Poloxamer } \\
\text { Ttriethanolamineoleate, } \\
\text { Capric triglycerides }\end{array}$ & Ultra-sonication & [51] \\
\hline Carbamazepine & Antiepileptic drug & Myristylmyristate Crodamo® & $\begin{array}{l}\text { Melt-emulsification } \\
\text { and ultra-sonication }\end{array}$ & [52] \\
\hline \multicolumn{5}{|c|}{ Hyperlipidemia } \\
\hline Atorvastatin Calcium & $\begin{array}{l}\text { HMG CoA enzyme } \\
\text { inhibitor/ } \\
\text { Hypolipidemic }\end{array}$ & $\begin{array}{l}\text { Poloxamer 188, Stearic acid, } \\
\text { Oleic acid, Tween-80, } \\
\text { Labrafac, } \\
\text { monostearate, Cutina Ply } \\
\text { Casein, } \\
\text { Groundnut oil, Precirol ATO } \\
5, \quad \text { Compritol 888ATO, } \\
\text { Gelucire 44/14, Linseed oil, }\end{array}$ & $\begin{array}{l}\text { High-pressure } \\
\text { homogenization }\end{array}$ & [53] \\
\hline
\end{tabular}




\begin{tabular}{|c|c|c|c|c|}
\hline Drug & Purpose & Excipients & Technique & Ref. \\
\hline & & $\begin{array}{l}\text { Caprylocaproyl macrogol-8- } \\
\text { glyceride }\end{array}$ & & \\
\hline Simvastatin & Hypolipidemic & $\begin{array}{l}\text { Glycerol monostearate, Oleic } \\
\text { acid, Poloxamer } 407\end{array}$ & Solvent injection & [54] \\
\hline \multicolumn{5}{|c|}{ Cancer } \\
\hline Tripertine & Anticancer & 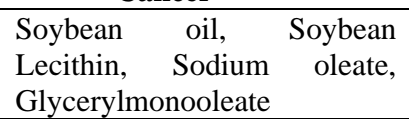 & Solvent-diffusion & {$[55]$} \\
\hline Turmeric powder & $\begin{array}{l}\text { Anticancer and anti- } \\
\text { inflammatory }\end{array}$ & $\begin{array}{l}\text { Medium-chain triglyceride oil, } \\
\text { Glycerol monostearate, Tween } \\
80\end{array}$ & Ultra-sonication & [56] \\
\hline Etoposide & $\begin{array}{l}\text { Chemo-therapeutic } \\
\text { agent }\end{array}$ & $\begin{array}{l}\text { Glyceryl monostearate, } \\
\text { Monostearin } \\
\text { distearoylphosphatidyl- } \\
\text { ethanolamine, Polyethylene } \\
\text { glycol } 40 \text { Stearate, Soybean } \\
\text { oil, Soya lecithin }\end{array}$ & $\begin{array}{l}\text { Low-temperature } \\
\text { solidification }\end{array}$ & [57] \\
\hline Paclitaxel & Lung cancer & $\begin{array}{l}\text { Stearic acid, Oleic acid, } \\
\text { Mannitol, Tween } 80 \text {, Tween } \\
\text { 20, Tween } 40\end{array}$ & Ultra-sonication & [58] \\
\hline \multicolumn{5}{|c|}{ Ocular disease } \\
\hline Curcumin & $\begin{array}{l}\text { Topical delivery in } \\
\text { ocular } \begin{array}{l}\text { fundus } \\
\text { pathologies }\end{array}\end{array}$ & 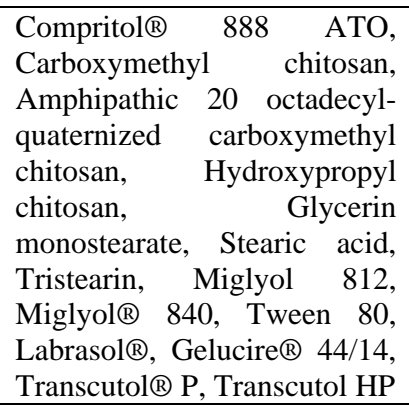 & Film-ultrasonic & [59] \\
\hline Ibuprofen & $\begin{array}{l}\text { Anti-inflammatory } \\
\text { for ocular drug } \\
\text { delivery }\end{array}$ & $\begin{array}{l}\text { Compritol ATO 888, Gelucire } \\
44 / 14, \quad \text { Miglyol 812, } \\
\text { Stearylamine, Transcutol }\end{array}$ & Melted-ultrasonic & {$[60]$} \\
\hline Flurbiprofen & $\begin{array}{l}\text { Anti-inflammatory } \\
\text { for ocular drug } \\
\text { delivery }\end{array}$ & $\begin{array}{l}\text { Compritol 888 ATO, Gelucire } \\
\text { 44/14, Miglyol 812N, Solutol } \\
\begin{array}{l}\text { HS-15, Chitosan } \\
\text { oligosaccharides }\end{array}\end{array}$ & Melt-ultrasonic & {$[61]$} \\
\hline $\begin{array}{l}\text { Triamcinolone } \\
\text { acetonide }\end{array}$ & $\begin{array}{l}\text { Corticosteroid as an } \\
\text { anti-inflammatory for } \\
\text { ocular drug delivery }\end{array}$ & $\begin{array}{l}\text { Ethylene oxide, propylene } \\
\text { oxide, Precirol®ATO5, } \\
\text { Squalene } ®, \text { Lutrol®F68, rac } \\
\text { 1-Oleoyl Glycerol }\end{array}$ & $\begin{array}{l}\text { High-pressure } \\
\text { homogenization }\end{array}$ & {$[62]$} \\
\hline \multicolumn{5}{|c|}{ Alopecia } \\
\hline Minoxidil & $\begin{array}{l}\text { Topical delivery for } \\
\text { treatment of alopecia }\end{array}$ & $\begin{array}{l}\text { Pluronic F-68, Carbopol } 934 \\
\text { Cholesterol, Soya lecithin, } \\
\text { Oleic acid, Tristearin, Tween- } \\
\text { 80, Triton X-100, } \\
\text { Triethanolamine }\end{array}$ & $\begin{array}{l}\text { Melt dispersion } \\
\text { ultra-sonication }\end{array}$ & [27] \\
\hline Spironolactone & $\begin{array}{l}\text { Follicular delivery for } \\
\text { alopecia }\end{array}$ & $\begin{array}{l}\text { Compritol }^{\circledR} 888 \text { ATO, Olive } \\
\text { oil, Tween } 80\end{array}$ & $\begin{array}{ll}\text { Emulsion } & \text { solvent } \\
\text { diffusion } & \\
\text { evaporation } & \\
\end{array}$ & [63] \\
\hline \multicolumn{5}{|c|}{ Hormone imbalance } \\
\hline Biochanin A & Phytoestrogen & 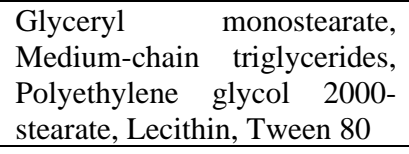 & $\begin{array}{l}\text { Emulsion- } \\
\text { evaporation and } \\
\text { low temperature- } \\
\text { solidification } \\
\end{array}$ & [64] \\
\hline \multicolumn{5}{|c|}{ Hepatoprotectant } \\
\hline Silymarin & Hepatoprotectant & $\begin{array}{l}\text { Lauroglycol 90, Labrafac PG, } \\
\text { Labrafac WL 1349, Labrafil } \\
\text { M } 1944 \text { CS, Precirol ATO 5, } \\
\text { Stearic acid, Capryol } 90\end{array}$ & $\begin{array}{l}\text { Emulsion- } \\
\text { evaporation }\end{array}$ & {$[65]$} \\
\hline \multicolumn{5}{|c|}{ Fungal disease } \\
\hline Itraconazole & Anti-fungal & $\begin{array}{l}\text { Compritol } 888 \text { ATO, Cetiol, } \\
\text { Precirol ATO 5, Almond oil, } \\
\text { Mygliol } 812 \text { N, Cutina CP, } \\
\text { Dynasan 114, Palmic acid, } \\
\text { Stearic acid, Witepsol E 85, } \\
\text { Cremophor EL, Cremophor }\end{array}$ & $\begin{array}{l}\text { Hot high-pressure } \\
\text { homogenization }\end{array}$ & {$[66]$} \\
\hline
\end{tabular}




\begin{tabular}{|c|c|c|c|c|}
\hline Drug & Purpose & Excipients & Technique & Ref. \\
\hline & & $\begin{array}{l}\text { RH 40, Eumulgin SLM 20, } \\
\text { Lutrol F68, Span } 85\end{array}$ & & \\
\hline \multicolumn{5}{|c|}{ Topical inflammation } \\
\hline Quercetin & $\begin{array}{l}\text { Anti-oxidation and } \\
\text { anti-inflammation for } \\
\text { topical delivery }\end{array}$ & $\begin{array}{l}\text { Glycerylmonostearate, Stearic } \\
\text { acid, Soya lecithin }\end{array}$ & $\begin{array}{l}\text { Emulsion } \\
\text { evaporation- } \\
\text { solidification } \\
\end{array}$ & [67] \\
\hline \multicolumn{5}{|c|}{ Lungs disorder } \\
\hline Montelukast & Bronchodilator & Precirol ATO-5, Capryol-90 & Melt emulsification & [68] \\
\hline \multicolumn{5}{|c|}{ Food and Nutraceutical } \\
\hline Alpha-lipoic acid & Antioxidant & $\begin{array}{l}\text { Glycerine monostearate, } \\
\text { Glyceryl triacetate }\end{array}$ & $\begin{array}{l}\text { High pressure } \\
\text { homogenization }\end{array}$ & [69] \\
\hline Pomegranate seed oil & Nutraceutical & $\begin{array}{l}\text { Compritol }{ }^{8} 888, \text { Glyceryl } \\
\text { behenate, Beeswax, Propolis } \\
\text { wax, Tween } 80 \text {, Lecithin }\end{array}$ & Ultra-sonication & [70] \\
\hline Rutin & Functional food & $\begin{array}{l}\text { Oleic acid, Citric acid, Tween } \\
80\end{array}$ & $\begin{array}{l}\text { Freeze drying } \\
\text { emulsification }\end{array}$ & [71] \\
\hline $\begin{array}{l}\text { Tristearin, high oleic } \\
\text { sunflower oil, } \beta \text { - } \\
\text { carotene }\end{array}$ & Functional Food & $\begin{array}{l}\text { Octadecanoic acid, Propylene } \\
\text { glycol monostearate }\end{array}$ & - & [72] \\
\hline
\end{tabular}

\section{Recent Patents on Nanostructured Lipid Carriers}

The important reasons for NLC's increased popularity and global successes are minimal regulatory barriers and the use of non-toxic, biodegradable, and biocompatible excipients like lipids and emulsifiers. All the components used are generally recognized as safe by regulatory authorities or have been approved for encapsulation of active compounds in pharmaceutical \& food. In either case, the utilization of all materials in an acceptable and approved range is indeed crucial. Most are acquired or comprise natural sources components within the human body, e.g., such as fatty acid and glycerol. These are well-tolerated and are known to minimize cytotoxic or detrimental drug reactions. In the last several years, lipid nanostructure carriers have been employed to investigate various therapeutic compounds. Table 3 gives a summary of patents in nanostructured lipid carriers.

Table 3. Recent patent based on nanostructured lipid carriers.

\begin{tabular}{|c|c|c|c|c|}
\hline Patent name & Patent number & Applicant & Publication date & Ref. \\
\hline $\begin{array}{l}\text { Nanostructured liposome vector } \\
\text { with highly effective antineoplastic } \\
\text { activity }\end{array}$ & CN101011358 & Zhejiang University & 08.08 .2007 & [73] \\
\hline $\begin{array}{l}\text { Silybin nanostructured lipid carrier } \\
\text { and preparation method thereof }\end{array}$ & CN101632638 & Shandong University & 27.01 .2010 & [74] \\
\hline $\begin{array}{l}\text { Coenzyme Q nanostructured lipid } \\
\text { carrier and preparation method } \\
\text { thereof }\end{array}$ & CN101658468 & $\begin{array}{l}\text { Suzhou Nanohealth } \\
\text { Biotech Co., Ltd. }\end{array}$ & 03.03 .2010 & [75] \\
\hline $\begin{array}{l}\text { Azithromycin nanostructured lipid } \\
\text { carrier and preparation method } \\
\text { thereof }\end{array}$ & CN101658493 & $\begin{array}{ll}\text { Suzhou } & \text { Nanohealth } \\
\text { Biotech Ltd. } & \end{array}$ & 03.03 .2010 & [76] \\
\hline $\begin{array}{l}\begin{array}{l}\text { Nanostructured lipid } \\
\text { preparation method, } \\
\text { application thereof }\end{array} \\
\end{array}$ & CN101890170 & $\begin{array}{l}\text { Shanghai University of } \\
\text { T.C.M. }\end{array}$ & 24.11 .2010 & [77] \\
\hline $\begin{array}{l}\text { Method for preparing a } \\
\text { nanostructured lipid carrier and a } \\
\text { product manufactured by the same }\end{array}$ & KR1020110137263 & Malaysian palm oil board & 22.12 .2011 & [78] \\
\hline $\begin{array}{l}\text { Nanostructured lipid carrier (NLC) } \\
\text { drug delivery systems for treatment } \\
\text { of neurodegenerative disorders }\end{array}$ & $\begin{array}{l}\text { IN1251/MUM/201 } \\
2\end{array}$ & Vikrant T. Kadam & 01.06 .2012 & [79] \\
\hline $\begin{array}{l}\text { Resveratrol nanostructured lipid } \\
\text { carrier and preparation method } \\
\text { thereof }\end{array}$ & CN102614091 & $\begin{array}{ll}\text { Xia } & \text { Qiang, } \\
\text { Zhao Wujun } & \end{array}$ & 01.08 .2012 & [80] \\
\hline $\begin{array}{l}\text { Nanoparticle formulations for skin } \\
\text { delivery }\end{array}$ & US20120195957 & $\begin{array}{l}\text { Sachdeva Mandip Singh, } \\
\text { Florida Agricultural and } \\
\text { Mechanical University } \\
\text { Patlolla Ram }\end{array}$ & 02.08 .2012 & [81] \\
\hline
\end{tabular}




\begin{tabular}{|c|c|c|c|c|}
\hline Patent name & Patent number & Applicant & Publication date & Ref. \\
\hline $\begin{array}{l}\text { Composite anti-screening } \\
\text { agent nanostructured lipid carrier } \\
\text { and preparation method thereof }\end{array}$ & CN102688152 & Southeast University & 26.09 .2012 & [82] \\
\hline $\begin{array}{l}\text { Nanotechnology-based herbal } \\
\text { composition for safe and effective } \\
\text { treatment of psoriasis }\end{array}$ & IN422/MUM/2011 & $\begin{array}{l}\text { Singh Kamalinder Kaur, } \\
\text { Patel Medha Chetan }\end{array}$ & 12.07 .2013 & [83] \\
\hline $\begin{array}{l}\text { Thymoquinone } \\
\text { loaded nanostructured lipid } \\
\text { carriers (tq-nlc) and uses thereof }\end{array}$ & MYPI 2012001818 & Universiti Putra Malaysia & 25.10 .2013 & {$[84]$} \\
\hline $\begin{array}{l}\text { Nanostructured lipid carrier loaded } \\
\text { with phenylethyl resorcinol, } \\
\text { preparation method thereof, and } \\
\text { cosmetic containing same }\end{array}$ & CN103860389 & $\begin{array}{lr}\text { Beinong } & \text { biochemical } \\
\text { (Suzhou Industrial Park) } \\
\text { Co., r r r } \\
\text { Suzhou } \quad \text { Nanohealth } \\
\text { Biotech Co., Ltd. } \\
\end{array}$ & 18.06 .2014 & {$[85]$} \\
\hline $\begin{array}{l}\text { Podophyllotoxin preparation } \\
\text { resisting condyloma acuminata } \\
\text { relapse and HPV latent infection }\end{array}$ & CN103893167 & $\begin{array}{lr}\begin{array}{l}\text { Nanfang } \\
\text { southern } \\
\text { university }\end{array} & \text { mospital of } \\
\end{array}$ & 02.07 .2014 & {$[86]$} \\
\hline $\begin{array}{l}\text { Quercetin nanostructured ripid } \\
\text { carrier and preparation method } \\
\text { thereof }\end{array}$ & CN104172184 & Southeast University & 03.12 .2014 & [87] \\
\hline $\begin{array}{l}\text { Psoralen-doxorubicin-loaded } \\
\text { composite nanostructured lipid } \\
\text { carrier preparation and preparation } \\
\text { method thereof }\end{array}$ & CN104367549 & Liaoning University & 25.02 .2015 & {$[88]$} \\
\hline $\begin{array}{l}\text { Idebenone lipid nanocarrier } \\
\text { composition for the treatment of } \\
\text { neurodegenerative disorders }\end{array}$ & IN276/MUM/2014. & Sachin Subhash Salunkhe & 11.09 .2015 & [89] \\
\hline $\begin{array}{lr}\text { Nanostructured Lipidic-polymeric } \\
\text { pharmaceutical } & \text { composition } \\
\text { encapsulating drugs } & \end{array}$ & IN2074/DEL/2014 & Panjab University & 29.01 .2016 & {$[90]$} \\
\hline $\begin{array}{l}\text { Nanostructured solid lipid carrier } \\
\text { coating vitamin A palmitate and } \\
\text { preparation method thereof }\end{array}$ & CN105496801 & $\begin{array}{l}\text { Shanghai Institute of } \\
\text { Technology }\end{array}$ & 20.04 .2016 & [91] \\
\hline $\begin{array}{l}\text { Hydrophilic modification asiatic } \\
\text { acid Nanostructured lipid carrier } \\
\text { and preparation method thereof }\end{array}$ & CN105919976 & $\begin{array}{l}\text { Zhejiang Academy of } \\
\text { Medical Sciences }\end{array}$ & 07.09 .2016 & {$[92]$} \\
\hline $\begin{array}{l}\text { ICAM-1 monoclonal antibody- } \\
\text { modified } \\
\text { simvastatin nanostructured lipid } \\
\text { carrier and preparation and } \\
\text { application }\end{array}$ & CN106074389 & Zhejiang University & 09.11 .2016 & {$[93]$} \\
\hline $\begin{array}{l}\text { N-acetyl-L-cysteine modified } \\
\text { curcumin Nanostructured lipid } \\
\text { carrier used for oral administration }\end{array}$ & CN106176677 & $\begin{array}{l}\text { China Pharmaceutical } \\
\text { University }\end{array}$ & 27.07 .2016 & [94] \\
\hline Topical nanodrug formulation & US15163724 & $\begin{array}{ll}\text { Hamidreza } & \text { Kelidari } \\
\text { Majid Saeedi } & \\
\end{array}$ & 26.01 .2017 & [95] \\
\hline $\begin{array}{l}\text { A nanostructured lipid carrier } \\
\text { encapsulates zingiber officinale oil }\end{array}$ & MYUI 2015002695 & $\begin{array}{ll}\text { Universiti } & \text { Teknologi } \\
\text { Malaysia } & \\
\end{array}$ & 02.05 .2017 & [96] \\
\hline $\begin{array}{l}\text { Nanostructured lipid carrier } \\
\text { modified by glycolipid polymer as } \\
\text { well as preparation method and } \\
\text { application of Nanostructured } \\
\text { lipid carrier }\end{array}$ & CN107115531 & Zhejiang University & 01.09 .2017 & [97] \\
\hline 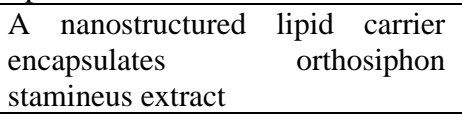 & MYPI 2016300023 & $\begin{array}{ll}\text { Universiti } & \text { Teknologi } \\
\text { Malaysia } & \end{array}$ & 29.06 .2018 & [98] \\
\hline $\begin{array}{l}\text { Cyclic peptide modified gambogic } \\
\text { acid Nanostructured lipid carrier } \\
\text { and preparation method thereof }\end{array}$ & CN108853054 & $\begin{array}{l}\text { Tianjin University of } \\
\text { Traditional Chinese } \\
\text { Medicine }\end{array}$ & 29.06 .2018 & [99] \\
\hline $\begin{array}{l}\text { Polymer thermosensitive liposome } \\
\text { loaded with yeast glucan and } \\
\text { carnosic acid }\end{array}$ & CN108904450 & $\begin{array}{l}\text { Guangzhou Jiayuan } \\
\text { Pharmaceutical } \\
\text { Technology Co., Ltd. } \\
\end{array}$ & 30.11 .2018 & [100] \\
\hline $\begin{array}{l}\text { Curcumin nanostructured lipid } \\
\text { carrier having improved heat } \\
\text { stability and efficient stabilization } \\
\text { heat treatment method thereof }\end{array}$ & KR1020190024397 & $\begin{array}{lr}\text { Industry } & \text { Academy } \\
\text { Cooperation } & \text { Foundation } \\
\text { of Sejong } & \text { University } \\
\text { Industry } & \text { Academy } \\
\text { Cooperation } & \text { Foundation } \\
\text { of Sejong University }\end{array}$ & 08.03 .2019 & [101] \\
\hline
\end{tabular}




\begin{tabular}{|c|c|c|c|c|}
\hline Patent name & Patent number & Applicant & Publication date & Ref. \\
\hline Ocular drug delivery & $\mathrm{WO} / 2019 / 123420$ & $\begin{array}{l}\text { Waterford Institute of } \\
\text { Technology }\end{array}$ & 27.06 .2019 & [102] \\
\hline $\begin{array}{l}\text { Nanostructured lipid carrier (NLC) } \\
\text { for collaborative treatment of } \\
\text { glioma as well as preparation } \\
\text { method and application of NLC }\end{array}$ & CN110013471 & $\begin{array}{l}\text { Stomatology/Affiliated } \\
\text { Stomatology Hospital of } \\
\text { Guangzhou } \quad \text { Medical } \\
\text { University }\end{array}$ & 16.07 .2019 & [103] \\
\hline $\begin{array}{l}\text { A Nanostructured solid lipid } \\
\text { carrier encapsulates bromelain } \\
\text { extract }\end{array}$ & MYPI 2018300001 & $\begin{array}{ll}\text { Universiti } & \text { Teknologi } \\
\text { Malaysia } & \end{array}$ & 22.07 .2019 & [104] \\
\hline $\begin{array}{l}\text { Novel nanostructured lipid carrier- } \\
\text { based ophthalmic controlled } \\
\text { release formulation for treatment in } \\
\text { fungal keratitis }\end{array}$ & IN201811021213 & $\begin{array}{lr}\text { Manish } & \text { Kumar } \\
\text { Ajay } & \text { Pathania } \\
\text { Vipin } & \text { Saini } \\
\text { A. } & \text { Pandurangan } \\
\text { Shailendra } & \text { Bhatt } \\
\text { Prerna Sarup } & \\
\end{array}$ & 13.12 .2019 & [105] \\
\hline $\begin{array}{l}\text { Nanostructured lipid carriers and } \\
\text { stable emulsions and uses thereof }\end{array}$ & EP3638207 & $\begin{array}{l}\text { Infectious Disease Res } \\
\text { Inst }\end{array}$ & 22.04 .2020 & [106] \\
\hline
\end{tabular}

\section{Conclusions}

Nanostructured lipid carriers have superior drug load capacity and tend to provide controlled drug release, which is attributable to the dissolution of the drug in oil and concurrently encapsulation in solid lipid. NLCs have acquired consistent prominence in recent times and shown considerable advantages in drug delivery versus traditional dosage formulations. The literature and patent review conducted in this review concluded that NLCs have a wide variety of pharmaceutical applications, including hypertension, diabetes, Parkinsonism, epilepsy, hyperlipidemia, cancer, alopecia, hormone deficiency, topical inflammation, ocular, hepatic, and fungal diseases. This study also explores NLC applications in the field of functional food and nutraceuticals.

\section{Current \& Future Developments}

Over the past decade, the number of studies describing formulations based on nanostructured lipid carriers has risen exponentially. The increase in the development of NLCs is majorly attributable to the surpassed barriers within the scientific formulation phase of lipidbased nanoparticles and improved awareness of the fundamental transport mechanisms of NLCs through various routes of administration. Due to its rapid absorption, bio-acceptability, and biodegradability, NLC is identified as a key drug delivery strategy without any alteration to the therapeutic agent. The influence of such delivery systems is steadily expanding and therefore has optimistic prospects for the future. It is indeed essential to further explore their application and effectiveness in food and pharmaceuticals.

\section{Funding}

This review received no external funding.

\section{Acknowledgments}

The authors express gratitude to Chitkara College of Pharmacy, Chitkara University, Punjab, India, for motivational support for this review's compilation.

\section{Conflicts of Interest}

The authors declare no conflict of interest. 


\section{References}

1. Pezeshki, A.; Hamishehkar, H.; Ghanbarzadeh, B.; Fathollahy, I.; Nahr, F.K.; Heshmati, M.K.; Mohammadi, M. Nanostructured lipid carriers as a favorable delivery system for $\beta$-carotene. Food Biosci. 2019, 27, 1117, https://doi.org/10.1016/j.fbio.2018.11.004.

2. Subramaniam, B.; Siddik, Z.H.; Nagoor, N.H. Optimization of nanostructured lipid carriers: understanding the types, designs, and parameters in the process of formulations. J. Nanopart. Res. 2020, 22, https://doi.org/10.1007/s11051-020-04848-0.

3. Salvi, V.R.; Pawar, P. Nanostructured lipid carriers (NLC) system: A novel drug targeting carrier. J. Drug Deliv. Sci. Technol. 2019, 51, 255-267, https://doi.org/10.1016/j.jddst.2019.02.017.

4. Duan, Y.; Dhar, A.; Patel, C.; Khimani, M.; Neogi, S.; Sharma, P.; Siva Kumar, N.; Vekariya, R.L. A brief review on solid lipid nanoparticles: part and parcel of contemporary drug delivery systems. RSC Adv. 2020, 10, 26777-26791, https://doi.org/10.1039/D0RA03491F.

5. Eleraky, E.N.; Omar, M.M.; Mahmoud, A.H.; Abou-Taleb, A.H. Nanostructured lipid carriers to mediate brain delivery of temazepam: Design and in vivo study. Pharmaceutics 2020, 12, https://doi.org/10.3390/pharmaceutics12050451.

6. Sarma, A.; Das, M.K. Formulation by design (FbD) approach to develop tenofovir disoproxil fumarate loaded nanostructured lipid carriers (NLCs) for the aptness of nose to brain delivery. J. Drug Deliv. Ther. 2019, 9, 148-159.

7. Djekic, L.; Martinović, M.; Ćirić, A.; Fraj, J. Composite chitosan hydrogels as advanced wound dressings with sustained ibuprofen release and suitable application characteristics. Pharm. Dev. Technol. 2020, 25, 332-339, https://doi.org/10.1080/10837450.2019.1701495.

8. Aljohani, M.; MacFhionnghaile, P.; McArdle, P.; Erxleben, A. Investigation of the formation of drug-drug cocrystals and coamorphous systems of the antidiabetic drug gliclazide. Int. J. Pharm. 2019, 561, 35-42, https://doi.org/10.1016/j.ijpharm.2019.02.024.

9. Sharma, M.; Sharma, R.; Jain, D.K.; Saraf, A. Enhancement of oral bioavailability of poorly water soluble carvedilol by chitosan nanoparticles: Optimization and pharmacokinetic study. Int. J. Biol. Macromol. 2019, 135, 246-260, https://doi.org/10.1016/j.ijbiomac.2019.05.162.

10. Singh, A.; Neupane, Y.R.; Mangla, B.; Kohli, K. Nanostructured lipid carriers for oral bioavailability enhancement of exemestane: Formulation design, in vitro, ex vivo, and in vivo studies. J. Pharm. Sci. 2019, 108, 3382-3395, https://doi.org/10.1016/j.xphs.2019.06.003.

11. Zhu, X.; Vo, C.; Taylor, M.; Smith, B.R. Non-spherical micro- and nanoparticles in nanomedicine. Mater. Horiz. 2019, 6, 1094-1121, https://doi.org/10.1039/C8MH01527A.

12. Jain, A.K.; Thareja, S. In vitro and in vivo characterization of pharmaceutical nanocarriers used for drug delivery. Artif. Cells Nanomed. Biotechnol. 2019, 47, 524-539, https://doi.org/10.1080/21691401.2018.1561457.

13. Czajkowska-Kośnik, A.; Szekalska, M.; Winnicka, K. Nanostructured lipid carriers: A potential use for skin drug delivery systems. Pharmacol. Rep. 2019, 71, 156-166, https://doi.org/10.1016/j.pharep.2018.10.008.

14. Tejashree, W.; Vamshi Krishna, R.; Srividya, G.; Ranendra Narayan, S.; Sunil Kumar, D.; Anu, P.; Gautam, S. Nanostructured lipid carriers as potential drug delivery systems for skin disorders. Curr. Pharm. Des. 2020, 26, 4569-4579, https://doi.org/10.2174/1381612826666200614175236.

15. Chauhan, I.; Yasir, M.; Verma, M.; Singh, A.P. Nanostructured lipid carriers: A groundbreaking approach for transdermal drug delivery. Adv. Pharm. Bull. 2020, 10, 150-165, https://dx.doi.org/10.34172\%2Fapb.2020.021.

16. D'Souza, A.; Shegokar, R. Nanostructured lipid carriers (NLCs) for drug delivery: Role of liquid lipid (oil). Curr. Drug Deliv. 2020, 18, https://doi.org/10.2174/1567201817666200423083807.

17. Nasiri, M.; Azadi, A.; Zanjani, M.R.S.; Hamidi, M. Indinavir-loaded nanostructured lipid carriers to brain drug delivery: Optimization, characterization and neuropharmacokinetic evaluation. Curr. Drug Del. 2019, 16, 341-354, https://dx.doi.org/10.2174/1567201816666190123124429.

18. Cernam, T.; Dinesh, S.; Narendar, D.; Nagaraj, B. Enhanced pharmacokinetic activity of zotepine via nanostructured lipid carrier system in wistar rats for oral application. Pharm. Nanotechnol. 2020, 8, 148160, https://dx.doi.org/10.2174/2211738508666200225113359.

19. Ranpise, H.A.; Gujar, K.N.; Pawar, S.C.; Awasthi, R.; Dua, K.; Mathure, D.; Madan, J.R. Formulation, optimization, and evaluation of ketoconazole loaded nanostructured lipid carrier gel for topical delivery. Drug Deliv. Lett. 2020, 10, 61-71, https://doi.org/10.2174/2210303109666190717155731.

20. Shirodkar, R.K.; Kumar, L.; Mutalik, S.; Lewis, S. Solid lipid nanoparticles and nanostructured lipid carriers: emerging lipid based drug delivery systems. Pharm. Chem. J. 2019, 53, 440-453, https://doi.org/10.1007/s11094-019-02017-9.

21. Pierre, A.H.; Mamdouh, M.G.; Shadeed, G. Development of betamethasone dipropionate-loaded nanostructured lipid carriers for topical and transdermal delivery. Antiinflamm. Antiallergy Agents Med. Chem. 2019, 18, 26-44, https://doi.org/10.2174/1871523017666181115104159.

22. Rizvi, S.Z.; Shah, F.A.; Khan, N.; Muhammad, I.; Ali, K.H.; Ansari, M.M.; ud Din, F.; Qureshi, O.S.; Kim, K.W.; Choe, Y.H.; Kim, J.K. Simvastatin-loaded solid lipid nanoparticles for enhanced anti-hyperlipidemic 
activity in hyperlipidemia animal model. Int. J. Pharm. 2019, 560, 136-143, https://doi.org/10.1016/j.ijpharm.2019.02.002.

23. Kamble, P.; Singh, P. A critical review on potential advancements of nanostructured lipid carriers in drug delivery. Curr. Nanomed. 2020, 10, 298-325, https://doi.org/10.2174/2468187310999200818110030.

24. Bhatt, S.; Kumar, M.; Devi, S.; Upadhyay, P.K.; Saini, V.; Mittal, A.; Mehan, N.; Saini, A. Recent advances in the development of the nanostructured lipid carriers for the topical fungal infections. J. Rep. Pharm. Sci. 2020, 9, https://doi.org/10.4103/jrptps.JRPTPS_99_19.

25. Haider, M.; Abdin, S.M.; Kamal, L.; Orive, G. Nanostructured lipid carriers for delivery of chemotherapeutics: A review. Pharmaceutics 2020, 12, https://doi.org/10.3390/pharmaceutics12030288.

26. Rathod, V.R.; Shah, D.A.; Dave, R.H. Systematic implementation of quality-by-design (QbD) to develop NSAID-loaded nanostructured lipid carriers for ocular application: preformulation screening studies and statistical hybrid-design for optimization of variables. Drug Dev. Ind. Pharm. 2020, 46, 443-455, https://doi.org/10.1080/03639045.2020.1724135.

27. Kochar, P.; Nayak, K.; Thakkar, S.; Polaka, S.; Khunt, D.; Misra, M. Exploring the potential of minoxidil tretinoin liposomal based hydrogel for topical delivery in the treatment of androgenic alopecia. Cutan. Ocul. Toxicol. 2020, 39, 43-53, https://doi.org/10.1080/15569527.2019.1694032.

28. Fernandes, A.V.; Pydi, C.R.; Verma, R.; Jose, J.; Kumar, L. Design, preparation and in vitro characterizations of fluconazole loaded nanostructured lipid carriers. Braz. J. Pharm. Sci. 2020, 56, https://doi.org/10.1590/s2175-97902019000318069.

29. Entezar-Almahdi E, Mohammadi-Samani S, Tayebi L, Farjadian F. Recent advances in designing 5fluorouracil delivery systems: a stepping stone in the safe treatment of colorectal cancer. Int. J. Nanomed. 2020, 15, https://dx.doi.org/10.2147\%2FIJN.S257700.

30. Poovi, G.; Vijayakumar, T.M.; Damodharan, N. Solid lipid nanoparticles and nanostructured lipid carriers: a review of the effect of physicochemical formulation factors in the optimization process, different preparation technique, characterization, and toxicity. Curr. Nanosci. 2019, 15, 436-453, https://doi.org/10.2174/1573413714666180809120435.

31. Li, T.; Nowell, C.J.; Cipolla, D.; Rades, T.; Boyd, B.J. Direct comparison of standard transmission electron microscopy and cryogenic-TEM in imaging nanocrystals inside liposomes. Mol. pharm. 2019, 16, 17751781, https://doi.org/10.1021/acs.molpharmaceut.8b01308.

32. Shaheen, M.A.; Elmeadawy, S.H.; Bazeed, F.B.; Anees, M.M.; Saleh, N.M. Innovative coenzyme Q 10loaded nanoformulation as an adjunct approach for the management of moderate periodontitis: preparation, evaluation, and clinical study. Drug Deliv. Transl. Res. 2020, 10, 548-564, https://doi.org/10.1007/s13346019-00698-z.

33. Kharat, M.; McClements, D.J. Fabrication and characterization of nanostructured lipid carriers (NLC) using a plant-based emulsifier: Quillaja saponin. Food Res. Int. 2019, 126, https://doi.org/10.1016/j.foodres.2019.108601.

34. Kadam, T.; Agrawal, S. A Short Review on the Important Aspects Involved in Preparation, Characterization and Application of Nanostructured Lipid Carriers for Drug Delivery. Curr. Nanomed. 2020, 10, 188-207, https://doi.org/10.2174/2468187310999200728140914.

35. Basha, S.K.; Dhandayuthabani, R.; Muzammil, M.S.; Kumari, V.S. Solid lipid nanoparticles for oral drug delivery. Mater. Today 2020, https://doi.org/10.1016/j.matpr.2020.04.109.

36. Lu, Y.; Mao, L.; Hou, Z.; Miao, S.; Gao, Y. Development of emulsion gels for the delivery of functional food ingredients: From structure to functionality. Food Eng. Rev. 2019, 11, 245-258, https://doi.org/10.1007/s12393-019-09194-z.

37. Abramov, E.; Ottaviani, M.F.; Shames, A.I.; Fattori, A.; Garti, N. Structural characterization of reconstituted bioactive-loaded nanodomains after embedding in films using electron paramagnetic resonance and selfdiffusion nuclear magnetic resonance techniques. Langmuir 2019, 35, 7879-7886, https://doi.org/10.1021/acs.langmuir.9b00578.

38. Bhella, D. Cryo-electron microscopy: an introduction to the technique, and considerations when working to establish a national facility. Biophys. Rev. 2019, 11, 515-519, https://doi.org/10.1007/s12551-019-00571-w.

39. Chockalingam, A. Impact of World Hypertension Day. Can. J. Cardiol. 2007, 23, 517-519, https://doi.org/10.1016/S0828-282X(07)70795-X.

40. Cryer, M.J.; Horani, T.; DiPette, D.J. Diabetes and Hypertension: A Comparative Review of Current Guidelines. The Journal of Clinical Hypertension 2016, 18, 95-100, https://doi.org/10.1111/jch.12638.

41. Atlanta, G.A. Centers for Disease Control and Prevention, U.S. Dept of Health and Human Services. National Diabetes Statistics Report, 2020.

42. Atlanta, G.A. Centers for disease control and prevention, U.S. Dept of Health and Human Services. National Diabetes Statistics Report, 2017.

43. Ranpise, N.S.; Korabu, S.S.; Ghodake, V.N. Second generation lipid nanoparticles (NLC) as an oral drug carrier for delivery of lercanidipine hydrochloride. Colloids Surf. B. Biointerfaces 2014, 116, 81-87, https://doi.org/10.1016/j.colsurfb.2013.12.012. 
44. Mishra, A.; Imam, S.S.; Aqil, M.; Ahad, A.; Sultana, Y.; Ameeduzzafar; Ali, A. Carvedilol nano lipid carriers: formulation, characterization and in-vivo evaluation. Drug Deliv. 2016, 23, 1486-1494, https://doi.org/10.3109/10717544.2016.1165314.

45. Nafee, N.; Makled, S.; Boraie, N. Nanostructured lipid carriers versus solid lipid nanoparticles for the potential treatment of pulmonary hypertension via nebulization. Eur. J. Pharm. Sci. 2018, 125, 151-162, https://doi.org/10.1016/j.ejps.2018.10.003.

46. Alam, T.; Khan, S.; Gaba, B.; Haider, M.F.; Baboota, S.; Ali, J. Adaptation of Quality by Design-Based Development of Isradipine Nanostructured-Lipid Carrier and Its Evaluation for In Vitro Gut Permeation and In Vivo Solubilization Fate. J. Pharm. Sci. 2018, 107, 2914-2926, https://doi.org/10.1016/j.xphs.2018.07.021.

47. Yin, J.; Hou, Y.; Yin, Y.; Song, X. Selenium-coated nanostructured lipid carriers used for oral delivery of berberine to accomplish a synergic hypoglycemic effect. International journal of nanomedicine 2017, 12, https://dx.doi.org/10.2147\%2FIJN.S144615.

48. Aslam, M.; Aqil, M.; Ahad, A.; Najmi, A.K.; Sultana, Y.; Ali, A. Application of Box-Behnken design for preparation of glibenclamide loaded lipid based nanoparticles: Optimization, in vitro skin permeation, drug release and in vivo pharmacokinetic study. J. Mol. Liq. 2016, 219, 897-908, https://doi.org/10.1016/j.molliq.2016.03.069.

49. Boushra, M.; Tous, S.; Fetih, G.; Xue, H.Y.; Tran, N.T.; Wong, H.L. Methocel-lipid hybrid nanocarrier for efficient oral insulin delivery. J. Pharm. Sci. 2016, 105, 1733-1740, https://doi.org/10.1016/j.xphs.2016.02.018.

50. Esposito, E.; Ravani, L.; Drechsler, M.; Mariani, P.; Contado, C.; Ruokolainen, J.; Ratano, P.; Campolongo, P.; Trezza, V.; Nastruzzi, C.; Cortesi, R. Cannabinoid antagonist in nanostructured lipid carriers (NLCs): design, characterization and in vivo study. Materials Science and Engineering: C 2015, 48, 328-336, https://doi.org/10.1016/j.msec.2014.12.012.

51. Esposito, E.; Mariani, P.; Ravani, L.; Contado, C.; Volta, M.; Bido, S.; Drechsler, M.; Mazzoni, S.; Menegatti, E.; Morari, M.; Cortesi, R. Nanoparticulate lipid dispersions for bromocriptine delivery: Characterization and in vivo study. Eur. J. Pharm. Biopharm. 2012, 80, 306-314, https://doi.org/10.1016/j.ejpb.2011.10.015.

52. Scioli Montoto, S.; Sbaraglini, M.L.; Talevi, A.; Couyoupetrou, M.; Di Ianni, M.; Pesce, G.O.; Alvarez, V.A.; Bruno-Blanch, L.E.; Castro, G.R.; Ruiz, M.E.; Islan, G.A. Carbamazepine-loaded solid lipid nanoparticles and nanostructured lipid carriers: Physicochemical characterization and in vitro/in vivo evaluation. Colloids Surf. B. Biointerfaces 2018, 167, 73-81, https://doi.org/10.1016/j.colsurfb.2018.03.052.

53. Khan, S.; Baboota, S.; Ali, J.; Narang, R.S.; Narang, J.K. Chlorogenic acid stabilized nanostructured lipid carriers (NLC) of atorvastatin: formulation, design and in vivo evaluation. Drug Dev. Ind. Pharm. 2016, 42, 209-220, https://doi.org/10.3109/03639045.2015.1040414.

54. Tiwari, R.; Pathak, K. Nanostructured lipid carrier versus solid lipid nanoparticles of simvastatin: Comparative analysis of characteristics, pharmacokinetics and tissue uptake. Int. J. Pharm. 2011, 415, 232243, https://doi.org/10.1016/j.ijpharm.2011.05.044.

55. Zhang, X.; Zhang, T.; Zhou, X.; Liu, H.; Sun, H.; Ma, Z.; Wu, B. Enhancement of Oral Bioavailability of Tripterine Through Lipid Nanospheres: Preparation, Characterization, and Absorption Evaluation. J. Pharm. Sci. 2014, 103, 1711-1719, https://doi.org/10.1002/jps.23967.

56. Park, S.J.; Garcia, C.V.; Shin, G.H.; Kim, J.T. Improvement of curcuminoid bioaccessibility from turmeric by a nanostructured lipid carrier system. Food Chem. 2018, 251, 51-57, https://doi.org/10.1016/j.foodchem.2018.01.071.

57. Zhang, T.; Chen, J.; Zhang, Y.; Shen, Q.; Pan, W. Characterization and evaluation of nanostructured lipid carrier as a vehicle for oral delivery of etoposide. Eur. J. Pharm. Sci. 2011, 43, 174-179, https://doi.org/10.1016/j.ejps.2011.04.005.

58. Kaur, P.; Garg, T.; Rath, G.; Murthy, R.S.R.; Goyal, A.K. Development, optimization and evaluation of surfactant-based pulmonary nanolipid carrier system of paclitaxel for the management of drug resistance lung cancer using Box-Behnken design. Drug Deliv. 2016, 23, 1912-1925, https://doi.org/10.3109/10717544.2014.993486.

59. Liu, R.; Wang, S.; Sun, L.; Fang, S.; Wang, J.; Huang, X.; You, Z.; He, X.; Liu, C. A novel cationic nanostructured lipid carrier for improvement of ocular bioavailability: Design, optimization, in vitro and in vivo evaluation. J. Drug Deliv. Sci. Technol. 2016, 33, 28-36, https://doi.org/10.1016/j.jddst.2016.03.009.

60. Li, X.; Nie, S.-F.; Kong, J.; Li, N.; Ju, C.-Y.; Pan, W.-S. A controlled-release ocular delivery system for ibuprofen based on nanostructured lipid carriers. Int. J. Pharm. 2008, 363, 177-182, https://doi.org/10.1016/j.ijpharm.2008.07.017.

61. Luo, Q.; Zhao, J.; Zhang, X.; Pan, W. Nanostructured lipid carrier (NLC) coated with Chitosan Oligosaccharides and its potential use in ocular drug delivery system. Int. J. Pharm. 2011, 403, 185-191, https://doi.org/10.1016/j.ijpharm.2010.10.013

62. Araújo, J.; Nikolic, S.; Egea, M.A.; Souto, E.B.; Garcia, M.L. Nanostructured lipid carriers for triamcinolone acetonide delivery to the posterior segment of the eye. Colloids Surf. B. Biointerfaces 2011, 88, 150-157, https://doi.org/10.1016/j.colsurfb.2011.06.025. 
63. Shamma, R.N.; Aburahma, M.H. Follicular delivery of spironolactone via nanostructured lipid carriers for management of alopecia. International journal of nanomedicine 2014, 9, https://dx.doi.org/10.2147\%2FIJN.S73010.

64. Wang, L.; Luo, Q.; Lin, T.; Li, R.; Zhu, T.; Zhou, K.; Ji, Z.; Song, J.; Jia, B.; Zhang, C.; Chen, W.; Zhu, G. PEGylated nanostructured lipid carriers (PEG-NLC) as a novel drug delivery system for biochanin A. Drug Dev. Ind. Pharm. 2015, 41, 1204-1212, https://doi.org/10.3109/03639045.2014.938082.

65. Piazzini, V.; Lemmi, B.; D’Ambrosio, M.; Cinci, L.; Luceri, C.; Bilia, A.R.; Bergonzi, M.C. Nanostructured lipid carriers as promising delivery systems for plant extracts: The case of silymarin. Applied Sciences 2018, 8, https://doi.org/10.3390/app8071163.

66. Pardeike, J.; Weber, S.; Haber, T.; Wagner, J.; Zarfl, H.P.; Plank, H.; Zimmer, A. Development of an Itraconazole-loaded nanostructured lipid carrier (NLC) formulation for pulmonary application. Int. J. Pharm. 2011, 419, 329-338, https://doi.org/10.1016/j.ijpharm.2011.07.040.

67. Chen-yu, G.; Chun-fen, Y.; Qi-lu, L.; Qi, T.; Yan-wei, X.; Wei-na, L.; Guang-xi, Z. Development of a Quercetin-loaded nanostructured lipid carrier formulation for topical delivery. Int. J. Pharm. 2012, 430, 292298, https://doi.org/10.1016/j.ijpharm.2012.03.042.

68. Patil-Gadhe, A.; Pokharkar, V. Montelukast-loaded nanostructured lipid carriers: Part I Oral bioavailability improvement. Eur. J. Pharm. Biopharm. 2014, 88, 160-168, https://doi.org/10.1016/j.ejpb.2014.05.019.

69. Wang, J.; Tang, J.; Zhou, X.; Xia, Q. Physicochemical characterization, identification and improved photostability of alpha-lipoic acid-loaded nanostructured lipid carrier. Drug Dev. Ind. Pharm. 2014, 40, 201-210, https://doi.org/10.3109/03639045.2012.753901.

70. Soleimanian, Y.; Goli, S.A.H.; Varshosaz, J.; Sahafi, S.M. Formulation and characterization of novel nanostructured lipid carriers made from beeswax, propolis wax and pomegranate seed oil. Food Chem. 2018, 244, 83-92, https://doi.org/10.1016/j.foodchem.2017.10.010.

71. Babazadeh, A.; Ghanbarzadeh, B.; Hamishehkar, H. Novel nanostructured lipid carriers as a promising food grade delivery system for rutin. J. Funct. Foods 2016, 26, 167-175, https://doi.org/10.1016/j.jff.2016.07.017.

72. Hentschel, A.; Gramdorf, S.; Müller, R.H.; Kurz, T. $\beta$-Carotene-Loaded Nanostructured Lipid Carriers. J. Food Sci. 2008, 73, N1-N6, https://doi.org/10.1111/j.1750-3841.2007.00641.x.

73. Fuqiang, H.; Yongzhong, D.; Hong, Y.; Jian, Y. Nanostructured liposome vector with highly effective antineoplastic activity. CN101011358, 2007.

74. Dianrui, Z.; Lejiao, J.; Huiling, C.; Cunxian, D.; Yancai, W.; Feifei, F. Silybin nano structured lipid barrier preparation method thereof. CN101632638, 2010.

75. Qiang, X.; Hongxia W. Coenzyme Q nano structured lipid carrier and preparation method thereof. CN101658468B, 2010.

76. Qiang, X.; Jiaying, W. Azithromycin nano structured lipid carrier and preparation method thereof. CN101658493, 2010.

77. Ying, L.; Nianping, F.; Zhiqiang, C.; Rong, T.; Shan, W. Nanostructured lipid carrier, preparation method and application thereof. CN101890170, 2010.

78. Ismal, R.; Hung, L.C.; Nang, H.L.L.; May, C.Y.; Basri, M.B.; Tejo, B.A. Method for preparing a nanostructured lipid carrier and a product manufactured by the same. KR1020110137263, 2011, URL.

79. Kadam, V.T.; Nanjwade, B.K. Nanostructured lipid carrier (NLC) drug delivery systems for treatment of neurodegenerative disorders. IN1251/MUM/2012, 2012, URL.

80. Qiang, X.; Wujun, Z. Resveratrol nanostructured lipid carrier and preparation method thereof. CN102614091, 2012.

81. Singh, S.M.; Ram, P. Novel nanoparticle formulations for skin delivery. US20120195957, 2012.

82. Qiang, X.; Xueyang, D. Composite anti-screening agent nanostructured lipid carrier and preparation method thereof. CN102688152A, 2012.

83. Kaur, S.K.; Chetan, P.M. Nanotechnology based herbal composition for safe and effective treatment of psoriasis. IN422/MUM/2011, 2013, URL.

84. Yazan, L.S.; Keat, N.W.; Abdullah, R.; Wun, H.C. Thymoquinone loaded nanostructured lipid carriers (TQNLC) and uses thereof. MYPI 2012001818, 2013, URL.

85. Qiang, X.; Yanling, C.; Hengfeng, F.; Yiqing, H.; Jun, H.; Tingting, Z.; Yi, Z.; Quin, C. Nanostructured lipid carrier loaded with phenylethyl resorcinol, preparation method thereof and cosmetic containing same. CN103860389A, 2014.

86. Kang, Z. Podophyllotoxin preparation resisting condyloma acuminata relapse and HPV latent infection. Nanfang Hospital of Southern Medical University. CN103893167A, 2014.

87. Qiang, X.; Shilei, N. Quercetin nanostructured lipid carrier and preparation method thereof. CN104172184A, 2014.

88. Tiange, C.; Yu, C.; Fengde, T. Psoralen-doxorubicin-loaded composite nanostructured lipid carrier preparation and preparation method thereof. CN104367549A, 2017.

89. Salunkhe, S.S.; Bhatia, N.M.; Bharia, M.S. Idebenone lipid nanocarrier composition for the treatment of neurodegenerative disorders. IN276/MUM/2014, 2015, URL.

90. Kaur, I.P.; Kakker, S.; Singla, A. Nanostructured lipidic-polymeric pharmaceutical composition encapsulating drugs. IN2074/DEL/2014, 2016, URL. 
91. Wanping, Z.; Xiaomei, Z.; Xiaozhi, L.; Haiyang, Z. Nanostructured solid lipid carrier coating vitamin A palmitate and preparation method thereof. CN105496801A, 2016.

92. Wang, S.; Zhang, Y.; Yin, L.; Liang, Z. Hydrophilic modification asiatic acid nanostructured lipid carrier and preparation method thereof. CN105919976, 2016.

93. Jiang, S.; Li S.; Du, Y. ICAM-1 monoclonal antibody-modified simvastatin nanostructured lipid carrier and preparation and application. CN106074389A, 2016.

94. Xiao, Y.; Yu, A.; Wu, Y.; Wang, Z.; Yu, F.; Jin, X. A kind of curcumin nano-lipid carrier that the n-acetylL-cysteine for oral administration is modified. CN106176677, 2016.

95. Hamidreza, K.; Majid, S.Topical nanodrug formulation. US20170020896, 2017.

96. Aziz, R.; Sabtu, R.; Arifffin, F.D.; Rosli, N.A.; Aziz, A.A.; Hasham, R. A. Nanostructured lipid carrier encapsulates zingiber officinale oil. MYUI 2015002695, 2017, URL.

97. Fuqiang, H.; Tingting, M.; Hong Y.; Ming, Y. A kind of glycolipid polymers modification nano structured lipid carrier and preparation method and application. CN107115531, 2017.

98. Hasham, R.; Suhaimi, S.H.; Rosli, N.A.; Aziz, R.; Sarmidi, M.R. A nanostructured lipid carrier encapsulates orthosiphon stamineus extract. MYPI 2016300023, 2018, URL.

99. Zhidong, L.; Rui, H; Yumei, W.; Bing, Z.; Jiawei, L.; Jiaxin, P.; Pan, G., Dongli, Q.; Nan, L. A kind of gambogicacid nano structured lipid carrier and preparation method thereof of cyclic peptide modification. CN108853054, 2018.

100. Wang, B.; Wang, Q.; Bai, L. A kind of polymer thermal sensitive liposome loading yeast dextran and carnosic acid. CN108904450, 2018.

101. Hong Geun, P.; Geun, P.; Ko Sang, H.; Sang, H.; Jo Hye, E.; Hye, E.; Kim Yeong, E.; Yeong, E. Curcumin nanostructured lipid carrier having improved heat stability and efficient stabilization heat treatment method thereof. KR1020190024397, 2019, URL.

102. Behl, G.; Kumari, S.; O'reilly, N.; O’donovan, O.; Mcloughlin, P.; Kent, D.; Fitzhenry, L.; Behhaenry, E. Ocular drug delivery. WO2019123420A1, 2019.

103. Xie, M.; Xu, M.; Chen, X.; Zhou M. A kind of nano structured lipid carrier and its preparation method and application of synergistic treatment glioma. CN110013471, 2019.

104. Sarmidi, M.R.; Mohamad, S.T.; Hasham, R.A nanostructured lipid carrier encapsulates bromelain extract. MYPI 2018300001, 2018, URL.

105. Pathania, A.; Kumar, M.; Sarup, P.; Bhatt, S.; Pandurangan, A.; Saini, V. Novel nanostructured lipid carrierbased ophthalmic controlled release formulation for treatment in fungal keratitis. IN201811021213, 2019, URL.

106. Fox, C.B.; Khandhar, A.P.; Van, H.N.; Erasmus, J.G.; Lin, S.S. Nanostructured lipid carriers and stable emulsions and uses thereof. EP3638207, 2020. 